\section{Predicting the effect of decompression sickness on survival following submarine tower escape}

\author{
Geoff A.M. Loveman and Joel J.E. Edney \\ Maritime Life Support, QinetiQ Haslar, Gosport, UK
}

Submarine tower escape

Received 27 July 2020 Revised 26 November 2020 Accepted 25 December 2020

\begin{abstract}
Purpose - The purpose of the present study was the development of a methodology for translating predicted rates of decompression sickness (DCS), following tower escape from a sunken submarine, into predicted probability of survival, a more useful statistic for making operational decisions.

Design/methodology/approach - Predictions were made, using existing models, for the probabilities of a range of DCS symptoms following submarine tower escape. Subject matter expert estimates of the effect of these symptoms on a submariner's ability to survive in benign weather conditions on the sea surface until rescued were combined with the likelihoods of the different symptoms occurring using standard probability theory. Plots were generated showing the dependence of predicted probability of survival following escape on the escape depth and the pressure within the stricken submarine.

Findings - Current advice on whether to attempt tower escape is based on avoiding rates of DCS above approximately $5 \%-10 \%$. Consideration of predicted survival rates, based on subject matter expert opinion, suggests that the current advice might be considered as conservative in the distressed submarine scenario, as DCS rates of $10 \%$ are not anticipated to markedly affect survival rates.

Originality/value - According to the authors' knowledge, this study represents the first attempt to quantify the effect of different DCS symptoms on the probability of survival in submarine tower escape.
\end{abstract}

Keywords Search and rescue, Decompression sickness, Sea survival, Submarine tower escape

Paper type Research paper

\section{Introduction}

In the event of a UK Royal Navy (RN) submarine being stranded on the seabed, evacuation is possible via a small airlock, the escape tower. The crew may be exposed to raised pressure within the distressed submarine (DISSUB) and subsequently in the escape tower. Escapers wear a Submarine Escape Immersion Suit (SEIS), which allows buoyant ascent to the surface. The associated rapid return to normal atmospheric pressure puts the crew at risk of suffering decompression sickness (DCS). On reaching the surface, the escaper inflates and boards a single-seat life raft that is supplied with the SEIS.

(C) QinetiQ Limited. Published by Emerald Publishing Limited. This article is published under the Creative Commons Attribution (CC BY 4.0) licence. Anyone may reproduce, distribute, translate and create derivative works of this article (for both commercial and non-commercial purposes), subject to full attribution to the original publication and authors. The full terms of this licence may be seen at http://creativecommons.org/licences/by/4.0/legalcode

The work was funded by the UK Ministry of Defence, Defence Equipment and Support organization and the Submarine Delivery Agency.

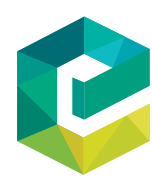

Journal of Defense Analytics and Logistics

Vol. 5 No. 1,2021 pp. $21-28$

Emerald Publishing Limited 2399-6439 DOI 10.1108/JDAL-07-2020-0014 
JDAL 5,1

If surface rescue assets are not yet on scene, surface survival following submarine escape will depend on weather conditions and on the physical state of the escapers, including any DCS symptoms suffered by the crew. Advice to the crew whether to attempt tower escape is contained within Guardbooks held aboard all classes of the UK RN submarine. The advice given currently instructs the crew not to attempt escape where the risk of any type of DCS is greater than an estimated $5 \%-10 \%$.

As the crew may not be familiar with the likely symptoms or consequences of DCS, information on probability of survival as opposed to probability of DCS would allow a better-informed decision to be made whether to attempt escape or remain on-board in anticipation of submarine rescue. This type of survival estimation is also used in modelling the effectiveness of various elements of the submarine escape, rescue, abandonment and survival system. Such analyses allow prioritisation of budget to provide the greatest predicted benefit in terms of lives saved.

A mathematical model for predicting the probability of DCS, $P(D C S)$, following submarine tower escape has been previously described (Loveman et al., 2014). The model was developed based on published methods (Weathersby et al., 1984; Thalmann et al., 1997). More recently, the methodology has been extended to allow the prediction of rates of four different types of DCS, classed by symptom as neurological, limb pain, respiratory (or cardiopulmonary) and cutaneous DCS (Edney et al., 2019). Through this extended methodology, four independent models were generated as follows:

- $\quad C N S 2 E E$ for the prediction of probability of neurological DCS, $P\left(D C S_{C N S}\right)$;

- Limb3LE, for probability of limb-pain DCS, $P\left(D C S_{\text {Limb }}\right)$;

- Resp1EE, for probability of respiratory DCS, $P\left(D C S_{\text {Resp }}\right)$; and

- Skin1EE, for probability of cutaneous DCS, $P\left(D C S_{S k i n}\right)$.

For any given pressure exposure, each model accepts a profile of changing ambient pressure with time and predicts the instantaneous risk of the relevant DCS symptom to the individual. The time integral of the instantaneous risk for each symptom, $r_{S}$, is transformed into a probability of DCS as shown in equation (1):

$$
P(P(D C S S))=1-e^{\int r_{S} \cdot d t}
$$

Equation (1) allows each of the four models to be used to predict the likelihood of each of the four selected types of DCS at any required time point following ascent to the surface.

The purpose of the present study was the development of a methodology for translating predicted $\mathrm{P}(\mathrm{DCS})$ into predicted probability of survival, $P($ Surv), a more useful statistic for making operational decisions.

\section{Methods}

Following ascent to the sea surface, DCS that occurs while in the water may prevent the survivor from boarding their single-seat life raft. For the purposes of this study, it was assumed that an individual unable to board their life raft would perish. Additionally, weather conditions were assumed to be benign. This implies that, in the absence of any form of DCS, a surfaced survivor who manages to board their single-seat life raft will remain alive sufficiently long to be located and picked up by surface forces and therefore to survive the scenario.

Should a submariner suffer DCS subsequent to having boarded their raft, they may perish in the raft because of the effects of the DCS, or they may remain alive sufficiently long 
to be picked up by surface forces, at which point it is assumed that medical treatment will be available and that they will survive the scenario.

Given the availability of the models for predicting the likelihood of the four selected types of DCS, then to determine the probability of survival, it remained to establish survival functions for the probability of:

- an individual boarding the single-seat life raft, for a given duration spent in the water, and given that DCS has not occurred;

- an individual boarding the single-seat life raft, for a given duration spent in the water and having suffered one, or a combination, of the four DCS categories; and

- continued survival in the single-seat life raft for a given duration after having suffered one, or a combination, of the four DCS categories.

As these survival functions cannot practicably be established through experiment, it was decided to use best estimates made by selected subject matter experts (SME) to arrive at usable values.

The pool of SME comprised five Surgeon Commanders from the RN Institute of Naval Medicine and one from the US Naval Medical Research Centre, all with backgrounds in diving and hyperbaric medicine and submarine medicine, and six researchers from QinetiQ Ltd., all of whom had extensive experience of hyperbaric trials in submarine escape, details of which have been previously published (Seddon et al., 2014; Jurd et al., 2014; Loveman et al., 2015).

For each survival function, probability estimates were made at time points of 5, 15, 30,60, 120, 240, 720 and 1,440 min. The estimated values are given in Tables 1-3.

\begin{tabular}{lc}
\hline Time in water since surfacing $(\mathrm{min})$ & Probability of boarding raft \\
\hline 5 & 0.99 \\
15 & 0.95 \\
30 & 0.8 \\
60 & 0.5 \\
120 & 0.2 \\
240 & $1 \mathrm{E}-18$ \\
720 & $1 \mathrm{E}-18$ \\
960 & $1 \mathrm{E}-18$ \\
1,440 & $1 \mathrm{E}-18$ \\
\hline
\end{tabular}

Table 1.

SME estimated survival function for probability of boarding raft within given time; values estimated by SME as having zero probability have been replaced with $1 \mathrm{E}-18$ to avoid computational errors

\begin{tabular}{|c|c|c|c|c|c|}
\hline $\begin{array}{l}\text { Time since DCS } \\
\text { occurred (min) }\end{array}$ & Neurological & Limb pain & Respiratory & Cutaneous & \\
\hline 5 & 0.8 & 1 & 1 & 1 & SME estimated \\
\hline 15 & 0.2 & 1 & 0.5 & 1 & survival functions \\
\hline 30 & $1 \mathrm{E}-18$ & 1 & $1 \mathrm{E}-18$ & 1 & determining effect of \\
\hline 60 & $1 \mathrm{E}-18$ & 1 & $1 \mathrm{E}-18$ & 1 & determining effect of \\
\hline 120 & $1 \mathrm{E}-18$ & 1 & $1 \mathrm{E}-18$ & 1 & suffering each DCS \\
\hline 240 & $1 \mathrm{E}-18$ & 1 & $1 \mathrm{E}-18$ & 1 & category on \\
\hline 720 & $1 \mathrm{E}-18$ & 1 & $1 \mathrm{E}-18$ & 1 & probability of \\
\hline 960 & $1 \mathrm{E}-18$ & 1 & $1 \mathrm{E}-18$ & 1 & boarding raft within \\
\hline 1,440 & $1 \mathrm{E}-18$ & 1 & $1 \mathrm{E}-18$ & 1 & given time \\
\hline
\end{tabular}


JDAL 5,1

Conferring between the SME was allowed and a consensus on each value was obtained through discussion or argument; such methods of judgement elicitation are usually referred to as "behavioural" (Rosqvist, 2003). follows.

Monte-Carlo techniques were adopted to determine the overall probability of survival as

The cumulative probability of each DCS symptom type at each time point was predicted using the four DCS symptoms models. These models have a dependency on body mass; a body mass of $80 \mathrm{~kg}$ was assumed here.

For each interval between two time points, $T_{n}$ and $T_{n+1}$, the probability, $P$, that an escaper would suffer a particular DCS symptom, given that they have not suffered that symptom so far, is:

$$
P(D C S s)=\frac{P\left(D C S s_{T n+1}\right)-P\left(D C S s_{T_{n}}\right)}{1-P\left(D C S s_{T n}\right)}
$$

A real value, $r$, in the range $0-1$ was randomly generated and compared with $P$, such that if $P>r$ then the escaper was assigned that particular symptom as having occurred. The first time at which the escaper suffered a given DCS symptom was stored, allowing duration of symptoms to be tracked.

For each time interval, the following probabilities were assigned according to similar logic, based on the relevant SME estimated survival functions:

- probability of boarding the single-seat life raft given the individual is still in the water and accounting for decreased likelihood of boarding with cumulative time in water and the effect of the tracked durations of any assigned combinations of DCS; and

- probability of perishing due to suffering, for the tracked durations, any assigned combinations of DCS symptoms.

Predicted events were assumed to be independent, such that the occurrence of any given event had no effect on the shape of the estimated survival function of other events. The probability of multiple events occurring was therefore taken as the product of their probabilities, in accordance with standard probability theory.

If the escaper was predicted to be alive in the raft after $24 \mathrm{~h}$, they were assumed to survive the scenario, regardless of any DCS symptoms. Any escaper still alive in the water after $24 \mathrm{~h}$ was assumed to perish at some time before rescue forces arrived; the effect of this assumption is indicated in the discussion.

Table 3.

SME estimated survival functions determining effect of suffering each DCS category on probability of survival in the life raft

\section{Time since DCS}

occurred (min)

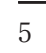

15

30

60

120

240

720

960

1,440

Neurological

Limb pain

Respiratory

Cutaneous

\begin{tabular}{lllll} 
& 1 & 1 & 1 & 1 \\
5 & 1 & 1 & 1 & 1 \\
0 & 0.9 & 1 & 1 & 1 \\
0 & 0.9 & 1 & 1 & 1 \\
20 & 0.9 & 1 & 1 & 1 \\
40 & 0.9 & 1 & 0.8 & 1 \\
20 & 0.8 & 1 & 0.1 & 1 \\
60 & 0.8 & 1 & 0.1 & 1 \\
140 & 0.8 & 1 & 0.1 & \\
\hline
\end{tabular}


This process was repeated for 100,000 simulated escapers, allowing estimation of $\mathrm{P}(\mathrm{Surv})$. Running 100,000 simulations was sufficient to establish the estimated value of P(Surv) to a tolerance of $0.1 \%$.

Also available from this method, at each time point, are the predicted proportion of the crew who are in their single-seat life raft or in the water, and the relative proportions of the crew who have no DCS or a combination of the four DCS categories.

For any given DISSUB scenario, the likelihood of the different DCS symptoms and the distribution of time of occurrence will vary with the pressure the crew are exposed to inside the submarine prior to escape and the depth from which escape is made. $\mathrm{P}(\mathrm{Surv})$ was estimated for a range of internal DISSUB pressures (1.0-3.0 bar) and DISSUB escape depths $(0-300 \mathrm{~m})$. Estimated $95 \%$ confidence intervals on predicted $\mathrm{P}($ Surv $)$ values were determined from the estimated confidence intervals of the original four DCS models using the method of propagation of errors $(\mathrm{Ku}, 1966)$.

\section{Results}

Table 1 shows SME anticipated that, in benign conditions, practically all surfaced survivors will be able to quickly board their single-seat life raft; submariners demonstrate their ability to do this during training. For the fraction of escapers who fail to board their raft immediately, the probability of boarding the raft was anticipated to fall to zero within the first $4 \mathrm{~h}$ of surfacing. This was argued to be due to exhaustion following repeated attempts to board the raft.

Table 2 shows that SME anticipated limb pain and/or cutaneous DCS would have no impact on an individual's ability to board their life raft, whereas neurological and/or respiratory DCS would have a marked impact and progressively reduce the probability of boarding the raft over time.

Table 3 shows that SME anticipated limb pain and/or cutaneous DCS would have no impact on an individual's ability to survive in the single-seat life raft, whereas neurological DCS would have some impact and respiratory DCS a greater impact, the presence of either of these latter symptom types progressively reducing the probability of surviving in the raft.

Plots of $\mathrm{P}($ Surv) following submarine tower escape are shown in Figure 1; a similar plot is shown in Figure 2 with 95\% confidence intervals determined from the estimated confidence intervals of the original four DCS models. The Figures show the effect that DCS is estimated to have on probability of surviving escape, to the point of rescue. It is assumed that rescue occurs at some time after $24 \mathrm{~h}$ and that any DCS symptoms that have not resulted in an individual perishing within $24 \mathrm{~h}$ will not have any bearing on the individual's continued ability to survive in the life raft after that time until they are rescued. It is important to note that other factors that might impact survival, such as temperature, sea state and the availability of food and potable water, are not included in these estimates and must be accounted for separately.

The narrow white triangular area at the bottom of each set of curves in Figures 1 and 2 defines the zone in which internal DISSUB pressure would be equal to or greater than the ambient sea pressure, an unlikely scenario but one which would make tower escape unachievable by current procedures.

\section{Discussion}

As might be anticipated, the survival curves shown in Figures 1 and 2 have broadly the same shape as the previously generated iso-risk curves for risk of DCS following tower escape (Figure 4 in Edney et al., 2019). 


\section{JDAL}

5,1

26

Figure 1.

Probability of survival curves for an $80 \mathrm{~kg}$ individual for escape following $24 \mathrm{~h}$ at raised DISSUB pressure
Figure 2.

Probability of survival curves for an $80 \mathrm{~kg}$ individual for escape following $24 \mathrm{~h}$ at raised DISSUB pressure (black); 95\% confidence regions for each contour (grey)
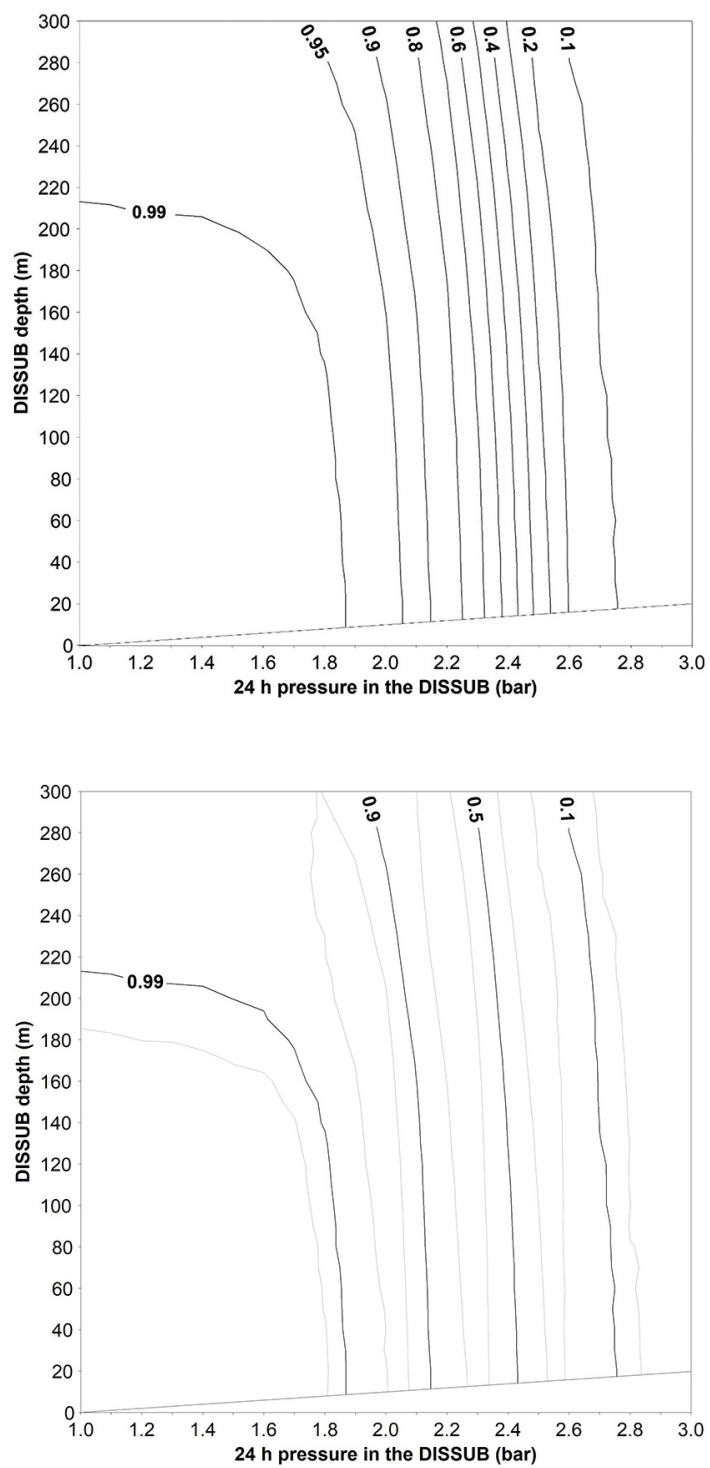

As the survival functions in Tables 1-3 were arrived at by SME consensus, this negated the possibility of using variance in experts' opinions to contribute to the estimated ranges on $P(S u r v)$ predictions. Thus, only the estimated error in the four DCS predictive models contributed to the $95 \%$ confidence regions as shown in Figure 2. For future risk estimation exercises, where consensus opinion is sought, it would be more useful either to obtain estimates of an agreed range of possible values for a given parameter or to collect individual or sets of estimates and use the (geometric) mean. 
Figures 1 and 2 as presented here are based on an assumption that the crew have spent $24 \mathrm{~h}$ at the ambient DISSUB internal pressure following the initial incident. This is sufficient time for their body tissues to become almost saturated with nitrogen; therefore, the figures may be regarded as valid for escape following $24 \mathrm{~h}$ or longer spent at the DISSUB pressure. It is possible to use the DCS models to generate predictions for any given duration spent in the DISSUB prior to escape. Tower escape occurring sooner than $24 \mathrm{~h}$ after the initial incident would be expected to result in lower rates of DCS, and predicted survival chances would therefore be better than those shown in Figures 1 and 2. However, overall survival rates may be increased by waiting for optimal surface conditions if this information is available to the crew. Non-benign surface conditions have not been considered here; the possible impact of surface weather on survival must be separately assessed. This could be done under an assumption of independence, or some effort could be made to account for possible potentiating effects of environmental factors on DCS. Inclusion of models of environmental factors in the survival prediction would allow inclusion also of the effect of the confidence intervals of predictions made with those models on the overall confidence of the combined survival prediction.

For the purposes of generating a single plot of survival curves, as shown in Figures 1 and 2, any escaper still alive in the water after $24 \mathrm{~h}$ was assumed to perish at some time before rescue forces arrived. It is worth noting that, even if DCS occurs, the probability of still being in the water at this time point is very low; this is due to the SME having assigned a high probability of boarding the raft immediately after the escaper surfaces, and the low probability of any DCS symptoms occurring within this time. So the overall effect of this assumption on predicted survival rates is small: In scenarios where the probability of DCS is low, the effect is negligible; where the predicted probability of DCS is high and therefore more personnel are unable to board their rafts, the effect is still small. For example, for an escape from $300 \mathrm{~m}$ and 3 bar internal DISSUB pressure, less than $0.8 \%$ of the crew are predicted to be in the water and alive at $24 \mathrm{~h}$. The predicted survival rate for this scenario is approximately $8 \%$, given that the prediction is based on SME estimates; the authors suggest it would be overly precise to conclude that the survival rate might be $8.8 \%$.

The DCS models used here do not include any representation of pulmonary barotrauma (PBT), that is, possible lung rupture due to expansion of air in the lungs as pressure reduces during ascent to the surface. PBT during the ascent phase of tower escape would likely result in death for most of those in which it occurs. PBT should not occur, provided that escapers do not hold their breath during ascent. However, since it has not been included here, the effect of PBT rate on survival would be to reduce the survival rate, approximately by the rate at which PBT occurs. A review by Benton et al. in 1999 showed that in 37,711 hooded ascents from $28.0 \mathrm{~m}$ in the Submarine Escape Training Tank (SETT), 27 incidents occurred that required some hospitalisation or recompression therapy or both (Benton et al., 1999), and at least six of these incidents resulted in unequivocal cases of pulmonary barotrauma. These data suggest the maximum rate of PBT events to be less than $0.1 \%$. It is possible that in an actual DISSUB incident, rates of PBT might be slightly higher, if escapers do not follow their training.

Current advice on whether to attempt tower escape is based on avoiding rates of DCS above approximately $5 \%-10 \%$. Consideration of predicted survival rates, based on SME opinion, suggests that the use of DCS rates in this way might be considered as conservative in the DISSUB scenario, as DCS rates of 10\% are not anticipated to markedly affect survival rates.

When applying probability theory, independence of events has been assumed. In reality, some of the events described here may not be independent of each other. For example, it might be anticipated that the physical exercise of repeated attempts to board the raft would 


\section{JDAL 5,1}

have some effect on the likelihood of DCS. As possible interaction effects are essentially unknown or unquantified, and also as SME opinion has been used in the absence of empirical data, the output presented here should be regarded and used in the understanding that it represents only the best guess that can be made at the present time.

The survival curves presented here are offered as guidance to submarine crews and rescue forces in preparation for, or in the event of, a DISSUB scenario. It must be noted that limitations in the use of these curves are similar to those previously described for the DCS models upon which the survival curves are based (Edney et al., 2019).

\section{References}

Benton, P.J., Francis, T.J.R. and Pethybridge, R.J. (1999), "Spirometric indices and the risk of pulmonary barotrauma in submarine escape training", Undersea and Hyperbaric Medical Society, Vol. 26 No. 4, pp. 213-217.

Edney, J.J.E., Loveman, G.A.M., Seddon, F.M., Thacker, J.C. and Jurd, K.M. (2019), "Prediction of signs/symptoms of decompression sickness following submarine tower escape", Undersea and Hyperbaric Medicine, Vol. 46 No. 1, pp. 17-33.

Jurd, K.M., Seddon, F.M., Thacker, J.C., Blogg, S.L., Stansfield, M.R.D., White, M.G. and Loveman, G.A.M. (2014), "Submarine 'safe to escape' studies in man", Undersea and Hyperbaric Medical Society, Vol. 41 No. 4, pp. 307-314.

Ku, H.H. (1966), "Notes on the use of propagation of error formulas", Journal of Research of the National Bureau of Standards, Section C: Engineering and InstrumentationVol. 70C No. 4, pp. 263-273.

Loveman, G.A.M., Seddon, F.M., Jurd, K.M., Thacker, J.C. and Fisher, A.S. (2015), "First aid oxygen treatment for decompression illness in the goat after simulated submarine escape", Aerospace Medicine and Human Performance, Vol. 86 No. 12, pp. 1020-1027.

Loveman, G.A.M., Seddon, F.M., Thacker, J.C., Stansfield, M.R. and Jurd, K.M. (2014), "Submarine tower escape decompression sickness risk estimation", Undersea and Hyperbaric Medical Society, Vol. 41 No. 4, pp. 315-329.

Rosqvist, T. (2003), "On the use of expert judgement in the qualification of risk assessment", Dissertation for degree of Doctor of Technology, VTT Technical Research Centre of Finland, Espoo, 14 October.

Seddon, F.M., Thacker, J.C., Fisher, A.S., Jurd, K.M., White, M.G. and Loveman, G.A.M. (2014), "Decompression illness in goats following simulated submarine escape: 1993-2006", Undersea and Hyperbaric Medical Society, Vol. 41 No. 4, pp. 301-306.

Thalmann, E.D., Parker, E.C., Survanshi, S.S. and Weathersby, P.K. (1997), "Improved probabilistic decompression model risk predictions using linear-exponential kinetics", Undersea and Hyperbaric Medical Society, Vol. 24 No. 4, pp. 255-274.

Weathersby, P.K., Homer, L.D. and Flynn, E.T. (1984), "On the likelihood of decompression sickness", Journal of Applied Physiology, Vol. 57 No. 3, pp. 815-825.

\section{Corresponding author}

Geoff A.M. Loveman can be contacted at: galoveman@qinetiq.com

For instructions on how to order reprints of this article, please visit our website: 